\title{
Transposition
}

Musique et Sciences Sociales

$7 \mid 2018$

Le prix de la musique

\section{Formes économiques et identité sociale des chœurs amateurs en France}

Guillaume Lurton

\section{(2) OpenEdition}

\section{Journals}

Édition électronique

URL : http://journals.openedition.org/transposition/1830

DOI : 10.4000/transposition. 1830

ISSN : 2110-6134

Éditeur

CRAL - Centre de recherche sur les arts et le langage

Référence électronique

Guillaume Lurton, «Formes économiques et identité sociale des chœurs amateurs en France», Transposition [En ligne], 7 | 2018, mis en ligne le 15 septembre 2018, consulté le 20 avril 2019. URL http://journals.openedition.org/transposition/1830; DOI : 10.4000/transposition.1830

Ce document a été généré automatiquement le 20 avril 2019

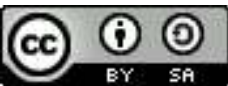

La revue Transposition est mise à disposition selon les termes de la Licence Creative Commons Attribution - Partage dans les Mêmes Conditions 4.0 International. 


\title{
Formes économiques et identité sociale des chœurs amateurs en France
}

\author{
Guillaume Lurton
}

1 L'amateurisme est souvent traité dans un rapport d'opposition frontale avec l'économie marchande. La dénégation de «l'intérêt commercial» est constitutive de la posture de l'amateur ${ }^{1}$ qui ne travaille pas pour le profit, mais « pour la gloire ${ }^{2}$ ». La pratique amateur n'aurait donc pas de prix. Le chant choral offre volontiers l'image d'un tel amateurisme "gratuit ", sans autre coût que l'engagement vocal spontané des individus. Chanter en chœur ne demande aucun investissement instrumental et peu de formation. Une telle légèreté économique a d'ailleurs contribué au développement du chant choral au cours $\mathrm{du} \mathrm{xx}^{\mathrm{e}}$ siècle. Le caractère démocratique d'une pratique musicale sans prix explique pour partie sa large mobilisation par les mouvements d'éducation populaire ${ }^{3}$.

2 La grille de lecture qui oppose l'économie des artistes professionnels à la gratuité de l'amateurisme est cependant trop simple. Si le chœur demande un investissement économique plus léger que la musique instrumentale, il n'en a pas moins un coût. Il implique la mobilisation de ressources matérielles (espace pour répéter et se produire, partitions, instruments accompagnateurs...) et de compétences (encadrement artistique et administratif du groupe). Les mécanismes qui permettent à des chœurs de mobiliser les moyens matériels de leur fonctionnement constituent l'économie du monde choral amateur. Cette économie est complexe. L'objectif de cet article est d'en proposer une description détaillée, afin d'élaborer une grille de lecture permettant de sortir de l'opposition entre professionnalisme marchand et amateurisme gratuit. Nous proposerons pour cela une typologie des modèles économiques sur lesquels repose l'économie chorale amateur, et nous montrerons que cette économie est "encastrée socialement »: la nature des mécanismes économiques mobilisés par les chœurs, varie selon l'identité sociale et artistique des groupes.

Méthodologie 
La description que nous proposons s'appuie sur approche ethnographique de l'économie chorale. La compréhension de ce monde amateur passe par une description fine des comportements économiques des acteurs. Nous nous appuyons pour cela sur des données à la fois qualitatives et quantitatives collectées dans le cadre de notre thèse de doctorat. Nous mobilisons des campagnes d'entretiens ${ }^{4}$ et d'observations ethnographiques réalisées au sein du monde choral amateur. Nous utilisons également des données statistiques collectées dans le cadre d'enquêtes quantitatives. Deux enquêtes par questionnaires de grande ampleur ont été réalisées au cours des années 2000 par les Missions Voix en Régions et par l'Institut Français d'Art Choral. La première réalise un état des lieux du chant choral. Elle a permis de collecter des données sur le fonctionnement global et le répertoire de 2451 chœurs $^{5}$. Les aspects économiques du fonctionnement de ces chœurs y sont évoqués à la marge. La seconde enquête porte sur un échantillon plus restreint de 681 chœurs $^{6}$, mais elle vise plus précisément les caractéristiques économiques de ces ensembles. Les faibles taux de réponse de ces enquêtes invitent à n'extrapoler les résultats qu'avec beaucoup de prudence. Ces données permettent néanmoins de saisir de grands équilibres et restent une source d'information précieuse sur les pratiques chorales amateurs.

5 Dans une première partie descriptive, nous dresserons le portrait de l'économie chorale amateur. Nous recenserons et décrirons les différents mécanismes sur lesquels repose le fonctionnement matériel des chœurs amateurs. Sur la base de cette approche empirique, nous dégagerons dans un second temps des clefs de lectures théoriques permettant de structurer notre vision de l'économie chorale amateur et d'expliquer les phénomènes observés.

\section{De la gratuité au prix de marché : les ressources du chœur amateur}

6 La gratuité d'une pratique amateur ne tient pas à l'absence de coût. Le fonctionnement de tout chœur génère des besoins matériels (hébergement, partitions...) auxquels sont apportées des solutions qui ne passent pas nécessairement par une évaluation chiffrée des coûts ni par la mobilisation de ressources monétaires. Certains groupes élaborent un budget leur permettant d'acheter les moyens matériels de leur fonctionnement et collectent les ressources monétaires nécessaires pour l'alimenter. D'autres se passent de monnaie et trouvent directement les moyens matériels de fonctionner.

7 Ces deux situations soulèvent deux questions différentes. Lorsqu'un chœur parvient à mobiliser des ressources sans que cela donne lieu à transfert monétaire, nous sommes dans une économie non monétaire qui peut justifier un sentiment de gratuité. Il s'agit alors de décrire comment un tel fonctionnement est possible. Quels sont les mécanismes permettant à un chœur de se passer de monnaie ? Lorsqu'un chœur a recours à un budget monétisé pour acheter les ressources dont il a besoin, l'enjeu descriptif change. Il s'agit alors de repérer d'où provient la monnaie mobilisée par le groupe, et de s'interroger sur ce qui rend cette mobilisation monétaire possible?

8 Nous organiserons notre description de l'économie chorale autour de cette articulation. Nous décrirons dans un premier temps les situations ou des chœurs satisfont leurs besoins sans avoir recours à la monnaie. Puis nous évoquerons les différentes sources de 
revenus monétaires susceptibles d'être mobilisées par les chœurs: Cotisations associatives, subventions, et produits de la vente de concerts.

\section{Illusions de la gratuité}

9 Les ressources mobilisées par un chœur pour fonctionner peuvent rester invisibles à l'échelle du groupe. $45 \%$ des chœurs interrogés sur leur fonctionnement économique ne déclarent aucun budget. Ces groupes ont pourtant besoin de lieux pour répéter, de partitions, d'instruments... Mais ces besoins n'entraînent aucun transfert monétaire visible à l'échelle du collectif. Un tel fonctionnement repose sur ce que Florence Weber décrit comme une «fiction de la gratuité $»^{7}$ : des ressources sont mobilisées sans que le groupe ne fournisse de contrepartie monétaire. Les coûts engendrés par le fonctionnement du collectif sont contournés ou reportés sur d'autres acteurs, individuels ou institutionnels. Le chœur reste, pour sa part, transparent sur le plan économique.

\section{Économies informelles}

10 La gratuité du chœur peut reposer sur un fonctionnement totalement informel. Jacques ${ }^{8}$ fait ainsi partie d'un quatuor vocal qui répète de façon hebdomadaire chez chaque chanteur à tour de rôle. Les membres du groupe apportent les partitions qu'ils ont récupérées dans d'autres chœurs, où qu'ils achètent à titre individuel. Ingrid pour sa part est musicienne intervenante. Elle anime des cérémonies religieuses marquantes au sein de sa famille ou de son cercle amical (mariages ou baptêmes). Elle monte de petites chorales liturgiques en mobilisant les bonnes volontés. Dans le cas de Jacques comme dans celui d'Ingrid, les petits chœurs évoqués donnent le sentiment d'une grande légèreté économique. Aucun coût n'est évoqué en entretien. De tels fonctionnements informels reposent sur trois types de ressources.

11 C'est en en premier lieu le patrimoine personnel des membres du groupe qui est mobilisé. Les partitions achetées par Jacques ont bien sûr une valeur marchande, mais leur propriétaire les met à disposition du groupe gratuitement. Les exemples de tels services rendus gracieusement par les membres de chœurs sont courants. Il est fréquent de voir un chef ou un chanteur apporter son clavier électrique pour accompagner la répétition. Et c'est jusqu'au principe central du monde amateur, le bénévolat, qui peut être considéré dans des termes équivalent : un individu met ses compétences au service du groupe sans en tirer de rémunération. La pratique du chant choral repose très largement sur une telle économie du don. Des chœurs informels peuvent fonctionner en tant que projet artistique collectif tout en reportant les coûts sur l'investissement de leurs membres. Le transfert de ressources en nature, des individus vers le collectif est une façon pour les chœurs de préserver l'illusion de la gratuité de la pratique chorale.

Une deuxième source de gratuité à laquelle puisent les chœurs est le domaine public. Les individus peuvent utiliser des "biens collectifs" sans avoir à s'acquitter de droits d'usage. Le domaine public est une ressource particulièrement importante en ce qui concerne le répertoire. L'interprétation d'œuvres est source de coût pour les chœurs amateurs, qu'il s'agisse de l'acquisition des partitions ou de l'acquittement de droits d'auteurs liés à la diffusion de l'œuvre en concert. Certains acteurs se tournent consciemment vers le répertoire des auteurs tombés dans le domaine public pour contourner ces coûts. Une choriste déclare ainsi avoir créé un ensemble vocal dont le 
répertoire est composé exclusivement de musique baroque sacrée. Elle relève incidemment à ce sujet que « ce qui est bien aussi, c'est qu'avec la musique baroque, il n'y a pas d'histoire avec la SACEM. » (Élisabeth, choriste)

Le coût principal lié au répertoire reste celui des partitions. Dans ce domaine, la question de la gratuité du domaine public n'est pas aussi simple qu'il y parait. Les droits de l'éditeur font également l'objet d'une protection. Et même si le compositeur est dans le domaine public, la reproduction de partitions peut rester illégale. Diverses pratiques visent à minimiser le coût des partitions tout en restant dans la légalité. Certains acteurs recopient des partitions manuellement ou à l'aide de logiciels d'édition musicale. Les sites internet proposant au téléchargement des partitions censées être libres de droits sont aussi fréquemment mobilisés par les acteurs du monde choral ${ }^{9}$.

En dehors du répertoire, la notion de domaine public peut être étendue à d'autres aspects du fonctionnement de chœurs informels. L'espace public en particulier est susceptible de servir de cadre à des pratiques chorales, répétitions ou concerts. Ingrid explique que les quelques répétitions du chœur qu'elle a monté pour animer la messe de mariage d'une amie, ont eu lieu dans le kiosque à musique d'un parc public parisien. Le quartet de Jacques donne des concerts de rue, en particulier lors de la Fête de la musique.

Une dernière solution permettant d'assurer la gratuité du fonctionnement d'un chœur consiste tout simplement à ne pas tenir compte des droits de propriété. Les acteurs du monde choral se mettent ainsi en marge de la légalité. Le respect du droit d'auteur et du droit de copie est particulièrement sujet à contournement. Le travail sur partitions photocopiées est extrêmement courant. Marianne dirige une petite chorale dont le fonctionnement est peu formalisé. Elle reconnaît spontanément en entretien que son chœur ne respecte pas les règles en la matière.

[Les partitions,] c'est tout photocopié Monsieur, c'est très très mal, mais je sais pas s'il y a beaucoup de chorales qui achètent des partitions. Nous on s'y met un peu, je sais pas si tu as vu qu'on a des originaux. [...] Après, s'il y a un contrôle, c'est clair qu'on est mal. (Marianne, chef de chœur)

Cette citation traduit une posture fréquente des acteurs du monde choral. S'ils sont réticents à l'idée d'acheter des partitions parfois couteuses, il ne s'agit pas non plus de se mettre intégralement hors-la-loi. On observe alors des positionnements ambigus : « c'est photocopié », mais « on s'y met un peu » (à acheter des originaux). La complexité du droit d'auteur favorise de telles ambiguïtés. Les pratiques de recours au domaine public évoquées plus haut sont sujettes à controverse et des acteurs sortent parfois du cadre légal sans en être pleinement conscients. L'un des principaux sites de téléchargement de partitions libres de droit se base ainsi sur le régime du Copyright Canadien et désengage sa responsabilité en signalant que les fichiers peuvent ne pas être dans le domaine public selon la législation du pays de l'usager. L'avertissement incite d'ailleurs les utilisateurs à recourir aux services d'un avocat spécialisé en droit de la propriété intellectuelle. Étant donnée la complexité de cette discipline juridique, de nombreuses pratiques se situent donc dans une zone grise. Les acteurs cherchent à minimiser le coût de leurs partitions tout en témoignant d'une volonté de rester au plus près de la légalité, plutôt que d'un strict respect de contraintes juridiques qui ne sont de toute façon pas maîtrisées.

\section{Chœurs attachés et aides en nature}

Les chœurs qui ne disposent d'aucun budget ne fonctionnent pas tous de manière informelle. Parmi les ensembles qui ne déclarent aucune ressource monétaire, la majorité 
(61\%) sont des chœurs "portés » dont le fonctionnement est pris en charge par une structure tierce mettant à disposition du chœur les moyens matériels nécessaires à son fonctionnement. Les coûts de la pratique chorale ne sont donc pas évalués à l'échelle du groupe. Ils sont intégrés à l'activité d'une organisation plus large qui l'englobe. La création d'un chœur universitaire, évoquée par son chef illustre bien ce type de situation. Le fonctionnement du chœur ne dépend plus de l'engagement matériel de ses membres, mais de celui de deux institutions.

[L'association régionale Musique et Danse a] proposé à l'université de fonctionner comme ça. La première année, ils payaient $60 \%$ du salaire du chef [...], l'université, 40. La deuxième année c'était le contraire, la troisième année, ils payaient plus que $10 \%$. Donc c'était une forte incitation puisque l'université ça lui coûtait pas cher au début. Mon travail est reconnu comme Unité de Valeur [à l'université]. (Pierre, Chef de chœur)

18 Cette citation illustre la particularité des chœurs concernés par ce mode de fonctionnement. Ces ensembles occupent une fonction précise au sein des structures auxquelles ils sont attachés. Comme ici, il s'agit en premier lieu de fonctions pédagogiques au sein d'institutions d'enseignement musical ou général (conservatoires, collèges, universités...). Il peut également s'agir de fonctions liturgiques pour les chœurs de paroisses, ou encore d'animation pour des activités chorales portées par des associations culturelles voire des chœurs d'entreprises...

La citation est représentative du type de dépenses prises en charge par les institutions qui portent des chœurs. Les chœurs portés par une institution se distinguent du reste du monde choral de par le statut de leurs chefs. Les données de l'enquête sur l'économie des chœurs aboutissent à un constat inattendu : il est très fréquent que les chœurs dirigés par des chefs professionnels déclarent ne disposer d'aucune ressource monétaire (aucune donnée budgétaire n'est renseignée). En réalité, parmi les ensembles dont le chef est rémunéré, les groupes indépendants qui budgétisent un salaire sont une minorité. Dans la plupart cas (62\% d'entre eux), c'est une structure tierce qui salarie le chef.

20 En dehors du salaire du chef de chœur, l'autre ressource fréquemment concernée par ce mode de fonctionnement est l'hébergement. La mise à disposition de locaux dépasse le cas des chœurs dont le fonctionnement est intégralement porté par une institution. Les répétitions de $88 \%$ des chœurs interrogés ont lieu dans un local mis à disposition gratuitement. Ces salles de répétitions sont des subventions en nature fournies par une municipalité ou une autre collectivité locale dans $57 \%$ des cas et un établissement d'enseignement général ou musical dans $28 \%$.

\section{Économies budgétaires}

21 Dans l'économie informelle, la vie économique du chœur se confond avec celle de ses membres. Dans l'économie des chœurs portés, elle se confond avec celle de l'organisation qui porte son fonctionnement. L'économie d'un chœur amateur sort de l'illusion de la gratuité et entre dans une économie monétaire à partir du moment où les ressources nécessaires à son fonctionnement sont évaluées et imputées au collectif, c'est-à-dire lorsqu'un budget de la pratique chorale est établi, et géré à l'échelle du groupe. Une telle formalisation est souvent motivée par la volonté des membres d'un chœur de dépasser les limites imposées par un fonctionnement purement informel, en affirmant l'existence du chœur en tant qu'entité autonome. Doter le chœur d'un budget revient alors à le constituer en tant que personne morale. En ce sens, le passage d'un fonctionnement non 
monétaire informel à une économie monétisée repose d'abord sur une transformation juridique. La très forte corrélation entre le fait de gérer un budget et le fait de disposer d'une identité juridique autonome (cf. tableau 1) souligne que ces deux phénomènes ne sont que deux versants d'un même problème.

\begin{tabular}{|c|c|c|c|c|}
\hline & \multicolumn{3}{|c|}{$\begin{array}{l}\text { Le chœur dispose-t-il en tant que tel de ressources } \\
\text { monétaires? }\end{array}$} \\
\hline & & Oui & Non & Total \\
\hline \multirow{2}{*}{$\begin{array}{l}\text { Le chœur a-t-il une } \\
\text { identité juridique propre? }\end{array}$} & Oui & $78 \%$ & $22 \%$ & $100 \%$ \\
\hline & Non & $16 \%$ & $84 \%$ & $100 \%$ \\
\hline \multicolumn{2}{|l|}{ Total } & $55 \%$ & $45 \%$ & $100 \%$ \\
\hline
\end{tabular}

Tableau 1

22 C'est dans le cadre de la forme associative que s'inscrit de façon quasi exclusive la construction de cette identité juridique des chœurs amateurs ${ }^{10}$. Fonder une association est une étape indispensable pour détacher la vie économique du groupe de celle de ses membres en le dotant des outils nécessaires : budget propre, compte en banque, moyens de paiement...

Dès lors qu'un groupe dispose des outils lui permettant de s'inscrire dans une économie monétaire, la mobilisation des ressources nécessaire à son fonctionnement change de nature. Il ne s'agit plus d'identifier les mécanismes lui permettant de capter des ressources matérielles, mais plutôt de comprendre la nature des ressources monétaires sur lesquelles repose la construction de son budget.

\section{Cotisations}

La cotisation est de loin le mode de financement le plus répandu: $88 \%$ des chœurs amateurs déclarent toucher des cotisations de la part des choristes. L'analyse de l'économie chorale associative passe par la compréhension de ce mode de financement. La cotisation associative est un échange: au transfert monétaire (paiement de la cotisation) répondent des contre-transferts en nature (remise de partitions au choriste, encadrement musical par le chef de chœur...). Cette transaction n'est pourtant pas un échange marchand. Elle ne présente aucune des caractéristiques qui permettent d'identifier la transaction de marché.

Une transaction marchande présente deux caractéristiques: le bien échangé est évalué indépendamment de la relation entre les personnes qui l'échangent; [...] c'est une relation fermée et affectivement neutre où transfert et contre-transfert se superposent, en principe instantanément, et épuisent le sens de l'interaction ${ }^{11}$.

L'évaluation du montant de la cotisation ne répond pas à ces critères. Ce montant ne résulte pas de la confrontation d'une offre et d'une demande anonymes, mais d'une relation personnalisée entre les partenaires de l'échange (le chœur et le choriste). La finalité de la cotisation est de mutualiser les coûts de la pratique entre les membres du groupe. Le montant de la cotisation reflète donc les caractéristiques du groupe : coûts 
engendrés et nombre d'adhérents. La façon dont Bernard évoque la fixation de la cotisation de son chœur illustre ce point.

Cette année elle est de $124 €$. [...] On a déjà demandé jusqu'à $150 €$. Cette année, il se trouve qu'on n'a pas prévu de week-end extérieur où il faut payer une pension complète. Donc la cotisation, on l'adapte tous les ans en fonction, on peut pas demander un fixe, parce que demander 150 cette année, ce serait voler le choriste par rapport au service qu'on lui offre. On essaye, tu vois, on l'a baissée, on l'a déjà augmentée. (Bernard, Chef de chœur)

Non seulement les caractéristiques du groupe sont essentielles dans la fixation de la cotisation, mais les caractéristiques individuelles des membres et en particulier leurs capacités de paiement entrent également en jeu. La mise en place de « tarifs » indexés sur la situation des personnes n'est pas rare dans le monde associatif. Le fait de demander une cotisation réduite aux jeunes, étudiants, chômeurs... traduit l'écart entre une relation marchande anonyme et la cotisation. Dans l'exemple évoqué par Sylvie, cette personnalisation va bien au-delà de la mise en place d'un « tarif », et devient une gestion au cas par cas négociée entre la trésorière et l'individu, puis validée par le bureau de l'association.

Quand il y a des personnes qui ont un peu des soucis d'argent, on essaye de s'organiser. Il y a une espèce de mutualisation [...]. On a une fille dans la chorale qui a pas trop trop de moyens, sa cotisation lui coûterait $120 €$, plus $35 €$ par week-end. Du coup ça fait plus de 200 et des brouettes, elle m'a dit « moi je peux pas débourser cette somme sur l'année, mais j'ai vraiment envie de faire partie de la chorale ». Donc je lui ai dit «combien tu peux mettre dedans? » Elle m'a dit 150, je lui ai dit ben d'accord. Et ça veut dire que les autres frais qu'elle va nous coûter vont être couverts par un petit pourcentage des cotisations des autres. Dans la mesure où ça met pas en péril les comptes de la chorale, moi ça me semble juste. (Sylvie, Trésorière)

La dernière phrase de cette citation résume en définitive les deux impératifs qui guident la fixation de la valeur de la cotisation. Il s'agit de ne "pas mettre en péril les comptes ", c'est-à-dire de garantir que le chœur pourra effectivement mobiliser les ressources dont il a besoin, sans creuser de déficit. De ce point de vue, l'économie chorale associative est une économie «budgétaire » au sens que Max Weber donne à ce terme ${ }^{12}$, c'est-à-dire une activité qui est sa propre fin et dont la seule contrainte est de parvenir à couvrir les coûts qu'elle engendre (par opposition à une économie lucrative dont la visée est de dégager un profit).

Le second impératif est de garantir une certaine « justice» au sein du groupe. L'échange qui se tisse autour de la cotisation s'écarte donc également de la deuxième caractéristique de la transaction marchande, dans laquelle la simultanéité du transfert et du contretransfert épuise le sens de la relation. L'impératif de justice renvoie au contraire à un horizon plus large : celui du fonctionnement du chœur. La formulation de cette trésorière de chœur associatif illustre ce point. Si la dimension économique de la cotisation est bien présente, elle se trouve relativisée (l'échange n'est pas qu'une transaction, mais également une prise de contact), et replacée dans un horizon temporel plus large: le paiement de la cotisation ne clôt pas la relation entre le choriste et le chœur, il en est au contraire le point de départ.

Récupérer les cotisations, c'est le travail du trésorier en début de saison. Et c'est pas anodin du tout. C'est un bon moment aussi pour prendre contact avec les membres de l'association [...]. C'était marrant parce qu'autour de cette petite transaction, tu vois qui n'a rien d'extraordinaire, ce qui est en jeu, c'est beaucoup plus que le fait de donner un chèque et d'en recevoir un. Ce qui est en jeu, ben c'est de se donner 
les moyens financiers de commencer l'année et d'avoir un peu de trésorerie.

(Élisabeth, Choriste, Trésorière de chœur associatif) de cotisations chorales. Dans la mesure où la cotisation est un échange personnalisé dans lequel les caractéristiques des partenaires rentrent en ligne de compte, la diversité des groupes et des situations individuelles débouche sur une grande diversité des montants de cotisations pratiqués par les chœurs amateurs. Les cotisations chorales peuvent s'élever d'un euro symbolique à plusieurs centaines d'euros ${ }^{13}$. Le caractère personnalisé de l'échange fait que le montant de la cotisation n'est pas soumis à la pression de mécanismes de marchés (le jeu de «l'offre et de la demande») qui pousseraient à l'uniformisation des montants : d'un point de vue économique, les chœurs ne sont pas en concurrence les uns avec les autres. Autrement dit, même si de nombreux choristes amateurs payent une cotisation pour pratiquer le chant choral, il n'existe ni marché ni prix de marché de la pratique chorale amateur.

\section{Subventions}

Les subventions constituent une seconde ressource financière pour les chœurs amateurs. Entre la moitié et les deux tiers des groupes amateurs touchent des subventions ${ }^{14}$. Le Tableau 2 donne un aperçu de la distribution de ces subventions en fonction des administrations dont elles émanent.

\begin{tabular}{|l|l|l|l|l|l|l|}
\hline & Commune & $\begin{array}{l}\text { Groupement de de } \\
\text { communes }\end{array}$ & $\begin{array}{l}\text { Conseil } \\
\text { Général }\end{array}$ & Région & DRAC & Autre \\
\hline Subvention médiane $(€)$ & 500 & 400 & 500 & 2000 & $\begin{array}{l}12 \\
000\end{array}$ & 400 \\
\hline Subvention moyenne $(€)$ & 1453 & 3982 & 2473 & 17651 & 471 & 5489 \\
\hline $\begin{array}{l}\text { Nombre d'ensembles } \\
\text { subventionnés }\end{array}$ & 306 & 26 & 129 & 27 & 15 & 51 \\
\hline $\begin{array}{l}\text { Proportion du nombre } \\
\text { total d'ensembles }\end{array}$ & $45 \%$ & $4 \%$ & $19 \%$ & $4 \%$ & $2 \%$ & $7 \%$ \\
\hline
\end{tabular}

Tableau 2

La distribution des subventions est marquée par une structure en chiasme. De l'échelle d'intervention publique la plus locale (les communes) à la plus large (les régions, et les crédits d'État déconcentrés des DRAC), le nombre d'ensembles aidés diminue alors qu'augmentent les sommes allouées. Près de la moitié des chœurs amateurs interrogés touchent des aides de leur commune d'implantation. Ils ne sont qu'un sur cinq à toucher des aides du département, et moins d'un sur vingt à toucher des aides de la région ou de la DRAC. Le montant de ces aides évolue de façon inverse : la subvention médiane passe de quelques centaines d'euros pour la commune et le département à plusieurs milliers pour la région. La même question se pose pour ces subventions que pour les cotisations associatives : quel sens accorder à ces aides? Selon quelle logique sont-elles versées aux 
chœurs amateurs? Deux logiques de distribution de subventions doivent être distinguées. L'une sanctionne l'animation de la vie associative d'un territoire, l'autre la qualité d'un projet artistique. Ce chargé de mission musique au sein d'un conseil général exprime bien cette opposition entre deux logiques de distribution d'aides aux associations chorales.

Chaque conseiller général dispose d'une enveloppe, et il est libre d'affecter cette enveloppe à qui il veut. Si c'est pour la foire au boudin, c'est pour la foire au boudin. Si c'est pour faire venir Boulez, c'est pareil. [...] Donc quand c'est d'intérêt local, ça s'appelle le fonds d'animation locale, ça dit bien ce que ça veut dire. Le critère, il est pas forcément de qualité artistique et culturelle, il est d'intérêt d'animation locale.

[...]

Éventuellement, on [le service culturel] aide les pratiques amateurs dans le cadre de projets, soit fédérateurs, qui présentent une dépense financière particulière et d'autre part un projet particulier... Je sais pas, une création, un concert exceptionnel avec orchestre... Mais à condition qu'il y ait un intérêt artistique, pas si c'est juste de dire "c'est notre anniversaire». (Chargé de mission musique, service culturel, conseil général)

Le premier programme d'aides géré directement par les conseillers régionaux dans le cas évoqué ici, ne cible pas les chorales en tant que structures artistiques, mais en tant qu'associations contribuant à l'animation sociale d'un territoire. Le second, géré par le service culturel, cible avant tout des projets comportant un contenu " artistique ». Cette distinction entre un critère "d'animation » et un critère de "qualité artistique » ne recouvre pas seulement une opposition dans la finalité de l'aide et dans les principes légitimant la subvention. Elle concerne également les formes que prend la distribution d'aides. Aux politiques de soutien à l'animation correspond une logique nondiscriminante. Ce n'est pas la nature de l'activité qui importe tant que son existence. Ce principe de subvention se traduit donc par la dispersion d'aides de faible montant parmi un nombre élevé de chœurs. Les subventions accordées en raison de la dimension " artistique » sont au contraire par nature discriminantes. Elles ciblent des projets en fonction d'un critère hiérarchisant de qualité esthétique. Ce principe de subvention tend à la concentration de subventions de montants élevés sur petit nombre de groupes.

\section{Production chorale : le prix du concert}

Les chœurs amateurs sont des producteurs artistiques. Ils construisent des programmes musicaux présentés à un public. Ils donnent en moyenne autour de 4 concerts par an. Pour la moitié d'entre eux, ces représentations génèrent des recettes - billetterie ou vente de concerts - qui constituent une troisième source de revenus monétaires. Dans quelle mesure la vente de leur production artistique inscrit-elle les chœurs dans une économie marchande?

\section{Le concert amateur, un don du public aux choristes}

Pour la plupart des chœurs amateurs, la relation économique qui se tisse entre un chœur amateur et son public est - comme pour la cotisation - une relation monétaire non marchande. L'anonymat qui définit la relation marchande fait à nouveau défaut. La composition du public des concerts amateurs est marquée par la très forte prééminence du réseau de proches des choristes. Interrogés à ce sujet, les membres de chœurs n'ont pas d'hésitation : le public appartient au réseau de connaissances des membres du chœur. 
Pour les concerts comme ça il faut pas se leurrer, le public est en grande majorité un public de famille, d'amis. Ce sont les musiciens, les choristes, enfin, c'est hyper difficile de capter un public qui est autre que ce public familial, amical. (Élisabeth, Trésorière)

Le public des chœurs amateurs n'a donc rien à voir avec une demande marchande anonyme. Les outils de communication dont disposent les chœurs expliquent cette situation. Seule une minorité de groupes amateurs ont recours aux outils de communication utilisés par les professionnels. $26 \%$ d'entre eux disposent de fonds d'affiche, $26 \%$ ont une plaquette, $30 \%$ ont d'un dossier de presse. La prévente de billets est en revanche assez largement répandue dans le milieu amateur : le public est recruté par les choristes qui vendent des billets à leurs proches dans les semaines qui précèdent le concert.

La familiarité avec le public peut se manifester de façon ostensible dans le déroulé même du concert. Les saluts de la main à un proche reconnu au sein du public en sont le signe le plus visible, mais pas nécessairement le plus fréquent. Plus subtils, mais plus présents sont les signes qui rompent l'impassibilité solennelle des interprètes en représentation expressions faciales, mimiques, manifestations de connivence entre des membres du chœur... Tel choriste n'est pas à sa place dans le rang des chanteurs qui entrent en scène. Il se replace dans la file, bousculant temporairement l'ordre de la cérémonie. Il a un haussement d'épaule gêné et une mimique qui semble signifier à la fois qu'il est désolé, et qu'il se sent un peu perdu. Tel autre écarquille les yeux face au public, surjouant la surprise de se trouver face à une assemblée aussi large... Si l'impassibilité du professionnalisme traduit l'anonymat de la relation entre l'interprète et son public, les mimiques et attitudes auxquelles nous faisons allusion transgressent cette perspective. Elles expriment un détachement vis-à-vis du formalisme de la situation et mettent à distance toute manifestation d'ambition qui pourrait être perçue par le public comme déplacée ou en décalage avec le niveau de la prestation. Les signes de connivence manifestés dans le courant du concert témoignent que la représentation ne découle pas de la recherche d'une reconnaissance de la qualité de la production artistique par le marché.

Le caractère non marchand de la billetterie de concerts a des implications économiques, tant sur la fixation du prix des billets que sur celle du nombre de concerts. Le caractère budgétaire de l'économie chorale amateur a des implications ici aussi. Les concerts sont des sources de dépenses importantes : location d'un lieu de représentation (salle, église... ), salaires et remboursements de frais à des intervenants extérieurs (instrumentistes accompagnateurs, solistes...)... Les responsables administratifs des chœurs amateurs sont attentifs à ce que les recettes occasionnées par le concert compensent autant que possible les dépenses. Il faut alors tenir compte de la capacité du chœur à capter un public de proches. Le raisonnement de ce chef de chœur à l'occasion d'une assemblée générale illustre ce point.

Au début de l'année, on avait pensé à faire un peu plus de concerts, deux concerts pour avoir un peu plus d'argent. Mais en fait, un seul concert c'est pas mal : les deux concerts de la chorale P... n'ont pas rempli, et avec les frais... (Antoine, chef de chœur, Assemblée générale du chœur A...)

La fixation du nombre de concerts et la fixation du prix ne relèvent donc pas d'un positionnement sur un marché, mais plutôt de considérations sur la disponibilité d'un public déjà connu et sur la capacité du chœur à rentrer dans ses frais. Dans la mesure où le public d'un chœur amateur est limité au cercle restreint des proches, la rentabilité 
marginale du concert est très vite décroissante et justifie le nombre limité de concerts organisés.

Si dans la forme, la vente de billets imite un échange marchand, dans les faits, l'échange qui se noue entre un chœur amateur et son public sort donc de ce cadre. Et c'est finalement le ressenti des choristes qui nous permet de mieux cerner la nature économique du concert amateur.

Par définition, à la fin de l'année, c'est un aboutissement, tout ce travail qu'on fait un peu à l'aveuglette, dans des salles de répètes, à l'esthétique plus ou moins moche, où on chante comme ça entre nous, en petit comité. On sait pas forcément trop où on va. Et d'un coup au concert, on met bout à bout des chants. Il y a même des gens qui les écoutent. [...] Les gens qui viennent nous voir, c'est les copains, c'est la famille, c'est des gens qui viennent pour nous faire plaisir. (Mathieu, choriste)

Ce qui marque dans cette citation, et qui est confirmé sans équivoque par l'observation des concerts du monde choral amateur, c'est le plaisir que tirent les choristes du concert. Mathieu souligne plusieurs fois au cours de l'entretien à quel point ce plaisir est un aboutissement important de son engagement choral. Une lecture marchande suggère qu'à travers la place de concert, le public achète une expérience esthétique. Dans la bouche de Mathieu pourtant, le public ne vient pas pour "se faire plaisir ", mais pour "nous faire plaisir ». Ce renversement conduit à relire le sens de la transaction: plus que l'achat d'une place de concert, le prix du billet d'entrée au concert amateur est un don du public aux membres du chœur, et ce à plus d'un titre. Il permet de financer l'organisation de la représentation tout en fournissant l'élément central du dispositif de concert : un public, qui de surcroit est acquis à la cause des artistes. Comme le précise Mathieu dans son entretien : « On sait qu'ils seront indulgents ».

\section{La marchandisation des concerts amateurs}

41 Pour une frange du monde choral amateur, le rapport au public sort de la relation de proximité que nous venons de décrire. Certains chœurs donnent un nombre important de représentations, pouvant aller jusqu'à plusieurs dizaines. Le cercle des proches ne suffit plus à remplir un nombre élevé de représentations, et la rentabilisation d'une telle diffusion passe par la mise en place de stratégies de captation d'un public élargi. Le chœur de Charles relève de ce cas de figure : la stratégie de diffusion mise en place vise avant tout à élargir l'audience.

Les premiers concerts, on les a faits gratuits, entrée libre, pour se former un public [...] On a fonctionné par e-mail essentiellement, et puis du tractage à la sortie des messes, à Sainte Clotilde, et des paroisses où on allait chanter, parce que c'est souvent dans des églises les concerts, des affiches. On a mis des annonces dans Zurban, l'officiel, le Pariscope, [...] On essaye de se faire une banque de données d'adresses e-mail, de gens qui ont envie d'être mis au courant de nos concerts, et qui commence à être assez fournie. (Charles, Président)

Cette transformation du rapport au public va souvent de pair avec une évolution du mode d'organisation des représentations. Les chœurs amateurs que nous avions évoqués organisent eux-mêmes leurs concerts, et les rentabilisent grâce à la billetterie. Les ensembles amateurs qui commencent à jouir d'une certaine réputation sollicitent - voire sont sollicités par - des organisateurs de concerts : collectivités locales, festivals, agents... Les chœurs qui parviennent à se constituer un réseau d'organisateurs de concerts prêts à 
faire appel à eux abandonnent peu à peu l'organisation de concerts, et se contentent de répondre aux sollicitations.

Il y a des gens qui nous téléphonent pour nous demander ce qu'on a en magasin, comme concert, on travaille beaucoup avec des municipalités. On est sollicité essentiellement par des organisateurs de concerts. Donc soit des municipalités, soit des structures, des agences. [...] On est très très peu à la recette, Une fois par an, on donne un concert, dans le cadre du festival où là on fait un concert à la recette, mais là on fait ça pour le fun. (Gérard, chef de chœur)

Le revenu tiré du concert change alors de nature. Il ne s'agit plus de recettes de billetteries, mais d'une vente de concert à un organisateur qui se chargera lui-même de la billetterie.

C'est $3000 €$ le concert. Bon, ce qui est pas non plus excessif. On connaît le tarif de certains chœurs professionnels, mais eux ils ont des frais, ils ont des charges, mais je pense qu'à 3000 , on est encore raisonnable. [...] Quand on fait un concert, l'église est toujours pleine. C'est aussi, la teneur de notre répertoire, de notre raison d'être, un chœur d'homme sur un [répertoire spécialisé], c'est suffisamment rare comme produit, effectivement un programmateur va pouvoir se dire, tient, si je mets un chœur comme ça, je suis sûr d'avoir du monde, et c'est vrai que ça se passe comme ça. Donc l'organisateur du concert, il va rentrer dans ses frais, ya pas de problème. (Michel, Chef de chœur)

Le prix n'est plus évoqué en référence aux frais engagés par le chœur dans une logique budgétaire. Le calcul entre dans une démarche de comparaison avec d'autres acteurs notamment professionnels qui conduisent ce chef à constater que « ce n'est pas excessif ». Cette logique comparative est le propre du positionnement sur une scène concurrentielle. La production de concert entre alors dans une logique marchande. Le vocabulaire utilisé (un "produit rare») marque également la rupture de logique économique. De tels ensembles, tout en restant bénévoles, entrent dans une logique marchande en se positionnant sur un marché des concerts.

\section{Les fondements sociaux de l'économie chorale}

Dès lors qu'on observe précisément la multiplicité des arrangements quotidiens qui permettent son fonctionnement, l'économie chorale apparait sous un jour nouveau: diversifiée, foisonnante, complexe. Elle échappe aux schémas explicatifs simples, et notamment à la seule notion de gratuité censée caractériser une économie de l'amateurisme. Sur la base de cette description, il s'agit donc dans cette deuxième partie de reconstruire un cadre explicatif permettant de comprendre les logiques économiques à l'œuvre dans le monde choral.

Ce tableau de la diversité des modes de fonctionnement d'une économie amateur soulève deux questions. La première, à visée descriptive, part du constat de la nature hétéroclite de l'économie chorale. Comment organiser ce foisonnement de modes de fonctionnement? Quels points de repère conceptuels peut-on mobiliser pour donner une lecture claire de cette économie? Nous commencerons donc par proposer des outils d'analyse permettant de structurer notre vision de l'économie du monde choral amateur.

La seconde, à visée explicative, porte le regard au-delà des seuls paramètres économiques pour réinscrire le fonctionnement matériel des chœurs amateurs dans le cadre plus large de leur vie sociale et artistique. Comment cette diversité des arrangements économiques du monde choral s'articule-t-elle avec les caractéristiques non économiques de ces 
groupes? Nous montrerons dans un second temps que la diversité de l'économie chorale n'est que le reflet de la diversité de ce que sont les pratiques chorales amateurs, d'un point de vue social et artistique.

\section{Typologies de l'économie chorale}

48 L'économie chorale amateur est extrêmement diverse. Pour mieux la comprendre, il faut baliser le paysage à l'aide de points de repère conceptuels. Nous nous appuierons pour cela sur les travaux de sociologie économique s'intéressant, dans la lignée de Karl Polanyi, à la diversité des "formes économiques ${ }^{15}$. Les théories de l'économie "plurielle ${ }^{16}$ » identifient trois formes d'organisation économiques : la réciprocité, la redistribution et le marché. Ces formes sont interprétées comme des secteurs économiques, définis par la nature des organisations qui y sont actives. Le secteur marchand est assimilé au monde de l'entreprise capitaliste, la redistribution au fonctionnement de l'État par le biais du prélèvement d'impôts permettant de délivrer des services publics, et la réciprocité enfin correspond à l'économie non monétaire de la sphère domestique et à l'activité des associations sans but lucratif ${ }^{17}$. Une telle façon d'envisager ce que sont les formes économiques ne permet pas de rendre compte de la diversité de l'économie chorale. Dans cette optique, le monde choral s'inscrirait exclusivement dans l'économie réciprocitaire $\mathrm{du}$ " tiers secteur ${ }^{18}$ ». Or, même s'ils relèvent effectivement d'une économie associative non-lucrative, tous les chœurs amateurs ne fonctionnement pas de la même façon.

Nous proposons donc de revenir à la typologie de Karl Polanyi en adoptant la démarche structurale qui est la sienne dans La Grande transformation. Il n'y définit pas des formes économiques par référence à des types d'organisation (Entreprises capitalistes, État et structures publiques, associations et sphère privée), mais en référence à la structure des réseaux sociaux qui portent les échanges économiques.

\section{Les formes de l'économie chorale}

L'économie chorale décrite en première partie est constituée de ressources en mouvement : des moyens matériels et monétaires circulent pour permettre aux groupes de fonctionner. Ces transferts de ressources reposent sur l'existence de réseaux d'acteurs économiques qui échangent. Comme toute économie, le fonctionnement matériel du monde choral dépend donc des relations qui existent entre acteurs, elle est " encastrée » dans des réseaux sociaux. Expliquer les formes de l'économie chorale signifie donc décrire les réseaux qui se tissent entre acteurs et qui permettent que des ressources soient allouées à la pratique chorale. La structure de ces réseaux définit des schémas de circulation des ressources, ou «formes économiques».

51 Les ressources économiques circulent selon un schéma de réciprocité lorsque ce réseau a une structure symétrique. On assiste à des échanges entre partenaires économiques de statut social équivalent. La forme paradigmatique de la réciprocité est l'échange nonmarchand de type don / contre-don. La réciprocité joue un rôle central dans l'économie chorale amateur. Elle peut-être non monétaire lorsque les ressources circulent directement en nature. C'est le cas notamment dans les économies informelles (mise à disposition gracieuse d'instrument, de partitions, d'espace de répétition...), et plus largement dans l'engagement amateur bénévole. La réciprocité peut également être monétaire. Nous avons vu que le prix du billet du concert amateur peut être assimilé à un 
don du public au chœur. Mais c'est surtout à travers la cotisation associative que la réciprocité joue un rôle fondamental dans le fonctionnement de l'économie chorale amateur. La cotisation n'est pas en effet un « prix de marché » de la pratique chorale. Elle est une ressource économique mise à disposition du groupe, qui permet au chœur de fonctionner, tout en marquant l'inclusion au collectif de l'individu qui s'en acquitte. La cotisation peut être lue comme un don fait par chacun aux autres membres du chœur. Elle peut être considérée comme une forme de réciprocité généralisée au sein du groupe.

Les ressources économiques circulent selon un schéma de redistribution lorsqu'il est possible d'identifier une entité centralisatrice - étatique ou non - qui polarise et régule la circulation des biens. Elle se distingue de la réciprocité par le fait que l'un des partenaires de la relation économique détient une autorité dans l'allocation des ressources. L'économie chorale repose pour une part importante sur des schémas redistributifs. Cela est évident dans le cas des subventions accordées par les tutelles publiques aux chœurs amateurs. La redistribution peut également s'opérer de manière non monétaire lorsque ces subventions sont accordées en nature (hébergement des répétitions, recrutement d'un chef de chœur professionnel), et plus largement lorsque le fonctionnement d'un chœur est porté par une autre organisation. Dans ce dernier cas, l'entité centralisatrice n'est plus nécessairement une tutelle publique. La centralisation découle de la structure hiérarchique de l'organisation qui gère le chœur parmi ses activités.

La notion de marché est définie de façon plus vague par Polanyi qui n'identifie pas de structure sociale propre à cette forme économique ${ }^{19}$. Nous nous appuierons sur la définition de Max Weber reprise par les travaux de sociologie des marchés ${ }^{20}$ qui en fait un échange dont les termes sont définis en situation concurrentielle. Il y a marché lorsque les acteurs économiques entrent en concurrence pour des "opportunités d'échange ${ }^{21}$ ", c'est-à-dire dès lors qu'interviennent des logiques de comparaison, et de négociation au cours desquelles les partenaires économiques anonymes se mettent d'accord sur les termes de l'échange qui leur semblent les plus avantageux. Alors même que cette logique concurrentielle semble extrêmement éloignée des principes de l'amateurisme, nous avons vu ressurgir la dépersonnalisation de la relation et la logique comparative qui sont au fondement de la forme marchande dans le cas des ensembles qui cherchent à élargir la vente de leurs concerts. Ces ensembles se rapprochent alors des modes de fonctionnement professionnels et sont évoqués comme « semi-professionnels » ou encore " grands-amateurs ».

Pour compléter ce tableau, signalons que des mécanismes marchands peuvent être observés marginalement sous une forme non monétaire. Un chœur hébergé par une paroisse déclare ainsi payer un loyer sous forme d'animations occasionnelles de l'office. Le nombre d'interventions est justifié par référence aux prix de marché d'une intervention liturgique du chœur et du loyer d'une salle de répétition.

On s'est arrangé avec le curé pour lui payer un loyer sous forme de prestations. On chante une messe par trimestre. Si on faisait payer, pour une messe de mariage, c'est environ $2000 €$. On n'en a jamais donné, mais quand je chantais à la Maîtrise, c'était ce tarif-là. Quatre fois, ça fait $8000 €$, c'est à peu près le prix d'une location. (Charles, Président d'association chorale).

L'échange donne bien lieu à la fixation d'une valeur d'échange qui s'apparente à une valeur marchande, sans faire intervenir d'équivalent monétaire. Dans la mesure où la transaction marchande dépend d'un accord sur la valeur de ce qui est échangé, la monnaie a vocation à y occuper une place centrale en tant qu'outil de mesure permettant 
de formuler cette équivalence. La référence aux prix monétisés dans cette citation révèle la difficulté de mener une négociation marchande indépendamment de l'outil monétaire. Il semble logique que la forme marchande non monétaire (le troc) reste marginale.

\section{Typologie et modèles économiques de chœurs amateurs}

Nous rencontrons donc dans l'économie chorale les trois formes économiques identifiées par Polanyi. Chacune peut être observée dans le cadre de transferts de ressources qui peuvent impliquer ou non l'usage de la monnaie. Du croisement de ces deux dimensions (caractère monétisé et structure des échanges) découle une typologie en six catégories (cf. tableau 3).

\begin{tabular}{|l|l|l|}
\hline & Non monétaire & Monétaire \\
\hline \multirow{2}{*}{$\begin{array}{l}\text { Réciprocité (structure sociale symétrique) } \\
\text { (structure sociale }\end{array}$} & $\begin{array}{l}\text { Eides et subventions en } \\
\text { nature } \\
\text { Bénévolat }\end{array}$ & $\begin{array}{l}\text { Économie } \\
\text { associative } \\
\text { cotisation } \\
\text { Concert amateur }\end{array}$ \\
\hline $\begin{array}{l}\text { Redistribution portés } \\
\text { centralisée) }\end{array}$ & $\begin{array}{l}\text { Transaction en nature (troc) } \\
\text { Exceptionnel }\end{array}$ & Marché du concert \\
\hline Marché (concurrence) & & \\
\hline
\end{tabular}

\section{TABLEAU 3}

57 Cette typologie est un outil descriptif du fonctionnement quotidien des chœurs amateurs à une échelle d'observation microsociale. Elle permet de caractériser les mécanismes que ces groupes mobilisent pour répondre à leurs besoins matériels. Un même ensemble peut tout à fait combiner plusieurs formes économiques. Un chœur associatif peut par exemple demander une cotisation pour acheter des partitions (réciprocité monétaire), répéter dans un local mis à disposition par la municipalité (redistribution non monétaire), organiser un "pot d'après concert» dont le buffet est préparé par les choristes (réciprocité non monétaire). À cette échelle, l'usage de la typologie met donc en évidence la souplesse d'une économie amateur qui combine une grande diversité de modes de fonctionnements. Mais elle permet d'ores et déjà de repérer certaines régularités dans la façon dont sont gérés les principaux enjeux matériels de la pratique chorale. Nous avons vu par exemple que les questions d'hébergement sont fréquemment pris en charge par la redistribution non monétaire (mise à disposition de salles par des municipalités ou des écoles), que les partitions font souvent l'objet d'une réciprocité, non monétaire (achat à titre individuel par les choristes et domaine public) ou monétaire (achats groupés par le chœur grâce aux cotisations), que la rémunération d'un chef de chœur repose le plus fréquemment sur la redistribution (prise en charge par une structure tierce dans le cadre d'un rattachement du chœur).

À l'échelle macro de l'ensemble du monde choral, la typologie permet de définir des «modèles économiques » qui décrivent cette fois le fonctionnement global d'un chœur. 
La façon dont certains chœurs mobilisent une forme économique de façon privilégiée, ou dont d'autres en combinent plusieurs, obéissent à certaines régularités. On voit émerger des équilibres récurrents dans la façon dont les chœurs organisent leur vie économique. Ces équilibres définissent des «modèles économiques». Ces modèles sont des idéaltypes qui permettent de caractériser l'identité économique d'un chœur. Nous en identifions quatre (cf. Tableau 4).

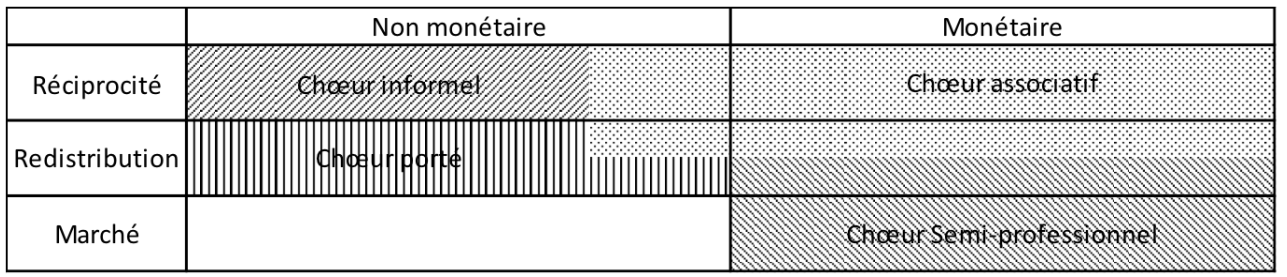

Tableau 4

Deux modèles économiques de chœurs amateurs reposent exclusivement sur des formes non monétaires. Il s'agit des chœurs informels et des chœurs portés, relèvent respectivement de mécanismes de réciprocité et de redistribution, et dont le fonctionnement a été décrit plus haut. Les chœurs associatifs sont un modèle plus souple dont le fondement est la réciprocité monétaire qui s'exprime en premier lieu à travers la cotisation. Ces ensembles associatifs combinent généralement plusieurs modes de fonctionnement en complément du budget alimenté par la cotisation. Ils peuvent mobiliser plus ou moins largement des mécanismes non monétaires (engagement matériel des individus et subventions en nature). Ils peuvent également s'appuyer sur des subventions, en particulier celles émanant des dispositifs de soutien à l'animation associative. Un dernier modèle économique est constitué par les ensembles que nous qualifions de semi-professionnels qui se distinguent par la façon dont ils diffusent largement leur production artistique, dans une logique marchande. Ces groupes peuvent toucher marginalement des cotisations, mais ils sont surtout susceptibles de toucher des subventions attribuées dans une logique de soutien à un projet artistique.

\section{Implications sociales et esthétiques des modèles économiques}

Il ne faut pas se tenir à une lecture purement économique des quatre modèles économiques que nous identifions. Nous l'avons souligné plus haut, l'économie des chœurs amateurs est «encastrée » socialement: elle dépend des relations sociales que tissent les acteurs impliqués dans leur fonctionnement (leurs membres, leur public, leurs partenaires...). Il existe donc un lien étroit entre ces modèles d'organisation économique et les autres composantes de leur fonctionnement: organisation sociale des groupes, manière dont se construisent les répertoires, organisation du travail artistique...

61 Il ne s'agit pas ici de donner une description exhaustive et détaillée des aspects non économiques de la vie des chœurs amateurs. Pour mettre en évidence l'encastrement social des modèles économiques des chœurs, nous insisterons sur un paramètre en particulier, déterminant pour comprendre l'économie des chœurs amateurs, à savoir les facteurs d'intégration sociale dont dépend la cohésion de ces groupes. Nous montrerons qu'à chaque modèle économique correspond un type d'intégration sociale particulier des chœurs amateurs. 


\section{Le prix de la gratuité, une forte intégration sociale} individuel. Il suppose un attachement au groupe fort de la part de ses membres. L'ampleur limitée de ces chœurs permet précisément une telle cohésion. Les individus peu nombreux, ou rassemblés pour un projet précis, entretiennent des liens étroits. Ils se connaissent souvent indépendamment de leur engagement choral. Dans le cas des chœurs d'Ingrid, il s'agit par exemple de réseaux amicaux et familiaux qui se rassemblent le temps d'une cérémonie religieuse. La cohésion de ces ensembles informels peut également tenir à des considérations esthétiques. C'est la motivation des membres du chœur pour un projet musical particulier qui garantit leur engagement, comme pour le quartet vocal de Jacques dont les membres sont rassemblés par un goût partagé pour le jazz vocal et la variété harmonisée.

65 Les chœurs portés ont souvent une plus grande ampleur que les chœurs informels. Les institutions porteuses ne fournissent pas seulement les ressources économiques nécessaires. Elles sont également des viviers de recrutement. Par ailleurs la nature de l'institution porteuse et le statut de la pratique chorale en son sein définissent plus ou moins explicitement l'identité du chœur porté : organe d'animation liturgique pour un chœur de paroisse, ensemble à vocation pédagogique pour un chœur de conservatoire, outil d'animation ou vecteur de cohésion pour des chœurs d'écoles, d'entreprise, de centres sociaux... Même si la finalité du chœur restait ambigüe, l'appartenance des individus à l'institution porteuse joue un rôle intégrateur.

Le facteur déterminant dans la définition d'un périmètre économique non monétaire est donc la solide cohésion des chœurs concernés. Ces groupes sont caractérisés par une forte intégration sociale, qui résulte d'un attachement des individus au collectif ou de l'emprise d'une institution sur le groupe. Ce constat conduit à reconsidérer le sens de la gratuité de l'amateurisme. Celle-ci est souvent lue comme le résultat de dispositions individuelles : désintéressement et vocation de l'amateur feraient passer l'art avant le profit. Nous 
faisons ici une lecture sociale de la gratuité, considérée comme un mode de fonctionnement collectif. Dans cette optique, c'est la forte intégration des groupes qui rend cette gratuité possible. Tous les chœurs amateurs n'ont pas une telle cohésion.

\section{La cohésion fragile des chœurs associatifs} énormément de marge aux individus dans la définition de leurs attentes vis-à-vis de cette activité. La maîtrise d'une technique musicale « classique » n'est pas indispensable et les compétences solfégiques des choristes amateurs peuvent être complètement absentes comme extrêmement poussées. Le répertoire est particulièrement vaste, et va des œuvres polyphoniques les plus savantes aux harmonisations de chanson populaire et de variété. Les motivations mêmes des choristes varient énormément. Certains évoquent une vocation artistique et mettent l'accent sur leurs attentes esthétiques. D'autres parlent du chant choral comme d'un loisir ou d'une activité sociale dans laquelle la dimension musicale du chœur est parfois anecdotique. La seule référence à l'identité chorale d'une association ne suffit donc pas à garantir la cohésion d'un chœur. À partir du moment où l'intégration sociale du collectif n'est plus garantie par l'attachement à un réseau de proches ou par l'appartenance à une institution, la cohésion des chœurs amateurs reste souvent fragile. Cette faible cohésion prend des formes variées, des oppositions de personnes bénignes, jusqu'aux fréquentes scissions de groupes qui rythment la vie du monde choral amateur.

69

La pérennité des chœurs associatifs dépend alors de mécanismes intégrateurs qui renforcent la cohésion du groupe dans le courant même de son fonctionnement. Alors que la cohésion forte des groupes non monétaires repose sur des mécanismes d'intégration indépendants de la pratique musicale (réseaux de sociabilités, institutions... ), la cohésion de ces chœurs associatifs se construit bon an mal an au fil de son fonctionnement quotidien. Dans l'ordre des choix esthétique par exemple, la multiplication de programmes éclectiques en est une manifestation des plus marquantes. Le mélange des esthétiques musicales les plus diverses est souvent un passage obligé pour les chefs de tels chœurs soucieux que "chacun s'y retrouve ». Le " plaisir du concert " que nous avons évoqué plus haut est un autre exemple de ces facteurs de cohésion qui tiennent au fonctionnement même du chœur amateur. Dans l'ordre économique, la cotisation est également l'un de ces mécanismes qui permettent d'entretenir la cohésion du groupe. Les termes utilisés par la trésorière associative citée plus haut doivent être relus dans cette optique : ce qui compte au moment du paiement de la cotisation, c'est la "prise de contact», et le fait de "se donner les moyens financiers de commencer l'année ». La cotisation est un geste économique qui «engage» le membre qui s'en acquitte. Cette dimension intégratrice de la cotisation associative est un reflet économique du besoin permanent des chœurs amateurs de renforcer leur cohésion. 


\section{La cohésion esthétique des chœurs semi-professionnels} marchande. À partir du moment où le chœur entre dans une logique concurrentielle, celle-ci impose une qualité de prestation qui fait peser une pression sur le groupe et ses membres. La valorisation marchande des concerts suppose que l'ensemble de ses membres aient un engagement fort, ce qui implique que tous partagent une conception relativement proche du sens de leur pratique chorale et de sa finalité artistique. La cohésion de ces groupes est donc en premier lieu esthétique. Alors que les répertoires musicaux éclectiques associant des styles très différents sont fréquents dans les chœurs associatifs, les ensembles semi-professionnels (ou "grands amateurs»), ont des répertoires généralement beaucoup plus ciblés. Pour beaucoup, cette spécialisation se fait sur des répertoires savants, mais elle peut également s'opérer sur les styles de musiques populaires : jazz vocal, variété harmonisée, répertoire choral traditionnel régional...

71 L'intégration esthétique a d'autres effets économiques. La capacité d'un groupe à mobiliser ses membres autour d'un projet artistique partagé favorise l'obtention de subventions ciblées sur la qualité esthétique. Nous avons vu que ces aides sont par nature plus discriminantes que les subventions accordées dans une logique d'animation, et tendent donc à concentrer des sommes plus importantes sur un nombre limité de groupe. Certains chœurs bien intégrés esthétiquement, dont les membres partagent une même vision de leur pratique chorale, peuvent donc avoir tendance à cumuler le revenu de la vente de leurs concerts et des subventions dont le montant est supérieur à la moyenne de celles des groupes amateurs. L'existence de cette frange de chœurs amateurs fortement intégrés sur le plan esthétique a donc des conséquences importantes sur la physionomie économique du monde choral. La capacité à capter conjointement des revenus marchands et des subventions creuse les écarts de moyens financiers entre chœurs amateurs.

Une telle intégration esthétique est rare dans le monde choral. Beaucoup d'individus chantent au contraire dans des ensembles dont ils ne partagent pas toutes les orientations esthétiques. L'affichage explicite d'un programme musical précis et d'exigences techniques n'est viable que si le groupe a déjà une bonne visibilité pour attirer suffisamment de chanteurs motivés. Pour des chœurs plus récents, l'intégration esthétique découle souvent, comme pour les chœurs informels, de liens que les membres $\mathrm{du}$ groupe entretiennent indépendamment $\mathrm{du}$ fonctionnement $\mathrm{du}$ groupe. Certains environnements sont particulièrement propices à la constitution de chœurs intégrés sur le plan esthétique: groupes d'étudiants en musicologie, d'élèves de conservatoires, musiciens se rencontrant lors d'un stage... Pour de tels ensembles, l'amateurisme peut d'ailleurs n'être que temporaire. De même que l'économie informelle n'est qu'une étape pour un groupe en cours de formation, l'économie « semi-professionnelle » n'est parfois qu'une étape sur la voie de la professionnalisation. De nombreux chœurs qui se sont professionnalisés entre les années 1980 et les années 2000 sont passés par cette phase de semi-amateurisme au cours de laquelle le groupe reste bénévole tout en se positionnant sur un marché du concert et en cherchant des aides afin de pérenniser leur fonctionnement. De telles trajectoires ont largement contribué à faire émerger le milieu choral professionnel qui s'est développé en France au cours des années 1980 et 1990.

Transposition, 7 | 2018 


\section{Conclusion}

73 L'opposition de la gratuité et du prix de marché ne suffit pas à définir l'économie de la pratique chorale amateur. Le monde choral amateur est complexe et son fonctionnement ne se laisse pas décrire de façon homogène par une seule logique économique. Il est au contraire traversé par des oppositions entre plusieurs modes de fonctionnement. Des mécanismes de réciprocité, de redistribution et de marché interviennent tant sous forme monétaire que non monétaire. Les six formes économiques qui découlent du croisement de ces deux dimensions définissent le répertoire des modes de fonctionnement économiques quotidien des chœurs amateurs. Quatre modèles types peuvent être identifiés pour définir la manière donc les ensembles se saisissent de ces formes économiques. Le fonctionnement des chœurs informels repose essentiellement sur la réciprocité non monétaire, celui des chœurs portés sur la redistribution non monétaire. Les chœurs associatifs combinent cotisations, subvention orientées vers l'animation associative, et revenus de concerts non marchands. Les ensembles semi-professionnels se distinguent par la valorisation marchande de leur production et leur capacité à capter des subventions attribuées aux projets artistiques. Ces modèles économiques sont encastrés dans la vie sociale du chœur. C'est l'intégration sociale forte des chœurs informels, ou le poids d'une institution porteuse, qui permet à certains ensembles de rester dans un modèle de gratuité, et c'est une forte intégration esthétique qui permet aux groupes semi-professionnels de se positionner sur un modèle marchand. La cohésion des chœurs associatifs reste en revanche fragile, et dépend de l'existence de mécanismes d'intégration dont la cotisation associative est un exemple dans l'ordre économique.

Les différents régimes économiques identifiés ne sont pas exclusifs. Certaines formes ne sont que temporaires et permettent l'évolution des groupes vers de nouveaux équilibres. Les chœurs informels ont souvent vocation à évoluer vers des groupes associatifs. Leur cohésion permet parfois l'émergence d'ensembles semi-professionnels qui eux même peuvent sortir de l'amateurisme en achevant de se professionnaliser. Ce constat nous incite donc à dépasser une vision des économies artistiques en termes de "mondes hostiles $^{22}$ ». L'économie du monde choral n'est pas faite de rupture ni d'antagonismes entre formes marchandes et gratuité. C'est au contraire la combinaison de ces modèles qui permet la fluidité de l'économie chorale.

\section{BIBLIOGRAPHIE}

BECKERT Jens, « The social order of markets », Theory and Society, $n^{\circ} 38,2009$, p. 245-269.

CUKROWICZ Hubert, « Le prix du beau », Genèses. Sciences sociales et histoire, ${ }^{n}$ O 36, 1999, p. 35-53.

DEL COL Éliane " "Travailler pour la gloire”. L'univers des oiseaux de cage », Genèses. Sciences

sociales et histoire, ${ }^{\mathrm{n}} \mathrm{O}$ 36, 1999, p. 6-34. 
EME Bernard et LAVILLE Jean Louis, « Économie plurielle, économie solidaire : précisions et compléments ", Revue du MAUSS, nº 7, 1996, p. 246-68.

FRANÇOIS Pierre, Sociologie des marchés, Paris, Armand Colin, 2008.

GoDBout Jacques, L'esprit du don, Montréal, Boréal, 1995.

GUMPLOWicz Philippe, Les travaux d'Orphée. Deux siècles de pratique musicale amateur en France, 1820-2000 : Harmonies-chorales-fanfares, Paris, Aubier, 2001.

LAVILLE Jean-Louis et SAINSAULIEU Renaud, Sociologie de l'association : des organisations à l'épreuve du changement social, Paris, Desclée de Brouwer, 2004.

LAVILLE Jean-Louis, «Le tiers secteur : un objet d'étude pour la sociologie économique », Sociologie du travail, $\mathrm{n}^{\circ} 42,2000$, p. 200.

POLANYI Karl, La grande transformation : aux origines politiques et économiques de notre temps, Paris, Gallimard, 2009.

STEINER Philippe, « Karl Polanyi, Viviana Zelizer et la relation marchés-société », Revue du MAUSS, ${ }^{\mathrm{n}} \mathrm{O} 29,2007$, p. $257-280$.

WEBER Florence, « Transactions marchandes, échanges rituels, relations personnelles ", Genèses, $\mathrm{n}$ ${ }^{\circ} 41,2000$, p. 85-107.

WEBER Florence, Le travail à-côté: une ethnographie des perceptions, Paris, Éditions de l'École des hautes études en sciences sociales, 2009.

WEBER Max, Economy and Society: An Outline of Interpretative Sociology, 2 vol., Berkeley, University of California Press, 1978.

\section{NOTES}

1. CUKRowicz Hubert, « Le prix du beau », Genèses. Sciences sociales et histoire, ${ }^{\text {no }}$ 36, 1999, p. 35-53.

2. DEL COL Éliane " "Travailler pour la gloire”. L'univers des oiseaux de cage ", Genèses. Sciences sociales et histoire, ${ }^{\mathrm{n}} \mathrm{O} 36,1999$, p. 6-34.

3. GUMPlowicz Philippe, Les travaux d'Orphée. Deux siècles de pratique musicale amateur en France, 1820-2000 : Harmonies-chorales-fanfares, Paris, Aubier, 2001.

4. Dans le cadre amateur du monde choral, 67 entretiens semi-directifs ont été réalisés, auprès de chefs de chœur, de chanteurs, mais également de responsables administratifs de structures associatives et d'acteurs impliqués dans l'encadrement politiques des pratiques chorales.

5. Le questionnaire avait été diffusé à l'intégralité des 5451 chœurs recensés par les quinze missions voix en région participant à l'enquête.

6. Le questionnaire avait été diffusé à un échantillon aléatoire de 3000 chœurs tirés parmi les bases de données des missions voix.

7. WEBER Florence, Le Travail à-côté: une ethnographie des perceptions, Paris, Éditions de l'École des hautes études en sciences sociales, 2009.

8. Les noms des individus interrogés en entretiens ont été modifiés.

9. Citons par exemple IMSLP Petrucci Music Library (International Music Score Library Project) et CPDL (Choral Public Domain Library).

10. $95 \%$ des chœurs disposant d'une identité juridique propre sont organisés en association « loi 1901 ». Les quelques ensembles restants sont pour la plupart des associations de droit local (en Alsace-Lorraine). 
11. WEBER Florence, «Transactions marchandes, échanges rituels, relations personnelles ", Genèse s, ${ }^{\text {n}} \mathrm{O} 41,2000$, p. 87

12. WEBER Max, Economy and Society: An Outline of Interpretative Sociology, 2 vol., Berkeley, University of California Press, 1978, p. 87-90

13. Sur l'échantillon interrogé, les montants vont de $1 €$ symbolique à $367 €$, la moyenne s'établissant à $42 €$.

14. Le taux varie de $63 \%$ dans l'enquête État des lieux à $53 \%$ dans l'enquête économie chorale.

15. POLANYI Karl, La grande transformation: aux origines politiques et économiques de notre temps, Paris, Gallimard, 2009.

16. EME Bernard et LAVILLE Jean Louis, «Économie plurielle, économie solidaire: précisions et compléments ", Revue du MAUSS, no 7, 1996, p. 246-68; LAVILLE Jean-Louis, "Le tiers secteur : un objet d'étude pour la sociologie économique », Sociologie du travail, no 42, 2000, p. 200.

17. Godbout Jacques, L'esprit du don, Montréal, Boréal, 1995.

18. LAVILLE Jean-Louis et SAINSAULIEU Renaud, Sociologie de l'association: des organisations à l'épreuve du changement social, Paris, Desclée de Brouwer, 2004.

19. Polanyi décrit précisément le marché comme une forme sociale "désencastrée ». Dans la mesure où l'échange marchand repose sur l'aboutissement d'une négociation entre deux partenaires dont la relation prend fin dès lors que l'échange est consommé, il considère que le marché ne repose sur la préexistence d'aucune structure sociale. Cet argument du désencastrement du marché étant très largement remis en cause par les acquis de la sociologie économique, nous ne retiendrons pas cette approche de l'économie marchande.

20. FRANÇOIS Pierre, Sociologie des marchés, Paris, Armand Colin, 2008 ; BECKERT Jens, "The social order of markets ", Theory and Society, ${ }^{\mathrm{n}} \mathrm{O} 38,2009$, p. 245-269.

21. WEBER, Economy and Society: An Outline of Interpretative Sociology.

22. STEINER Philippe, «Karl Polanyi, Viviana Zelizer et la relation marchés-société », Revue du MAUSS, ${ }^{\mathrm{n}} \mathrm{O} 29,2007$, p. 257-280.

\section{RÉSUMÉS}

La pratique musicale amateur a-t-elle un prix ? Spontanément, la légèreté économique du chant choral semble illustrer les représentations qui opposent la gratuité de l'amateurisme au prix fixé par le professionnalisme. Pourtant, si le chant choral amateur donne volontiers l'illusion de la gratuité, il n'en a pas moins un coût et il génère une économie plus complexe qu'il n'y paraît. Cet article établit une description ethnographique du fonctionnement économique des chœurs amateurs en France et propose des clefs de lecture pour en comprendre la logique. Nous proposons dans cette optique une typologie permettant de rendre compte d'une économie amateur. Celle-ci est encastrée socialement, le modèle économique d'un chœur amateur étant déterminé par son identité sociale et artistique.

\section{INDEX}

Mots-clés : chant choral, chœur, amateurisme, économie plurielle, marché, formes économiques non marchandes, économie non-monétaire, encastrement 


\section{AUTEUR}

\section{GUILLAUME LURTON}

Sociologue, Guillaume Lurton est maître de conférences à l'Institut d'Administration des Entreprises de l'Université de Poitiers. Ses travaux portent notamment sur le développement du chant choral en France et se positionnent à l'articulation entre sociologie de la culture et sociologie économique. Il s'intéresse en particulier à la diversité des formes économiques et à l'articulation entre marchés et formes économiques non marchandes. 